Bangladesh J. Pl. Breed. Genet., 28(1): 01-07, 2015

\title{
GENETIC DIVERSITY AMONG MAINTAINER LINES OF RICE (Oryza sativa L.) BASED ON CLUSTER ANALYSIS
}

\author{
M. J. Hasan, U. Kulsum, A. K. M. Shamsuddin ${ }^{1}$ and M. S. Islam \\ Hybrid Rice Division, Bangladesh Rice Research Inistitute, Gazipur 1701, Bangladesh \\ ${ }^{1}$ Department of Genetics and Plant Breeding, Bangladesh Agricultural University, Mymensingh \\ 2202, Bangladesh
}

\begin{abstract}
An experiment was conducted at the experimental field of Bangladesh Rice Research Institute (BRRI), Gazipur during T. Aman season 2007. Ten maintainer lines were evaluated to identify most diverse maintainer lines in respect of morphological traits for developing heterotic rice hybrids based on clustering and days to maturity. Based on mean values of different morphological traits and maturity, 10 maintainer lines were classified into four distinct clusters. Maximum Euclidean distance from centroids was observed in cluster 2 whereas cluster 3 and 4 found in the centroids. Most of the maintainer lines were grouped into early and medium maturing clusters while the remaining two lines were clustered into very early and late maturing clusters. Very early maturing group contained maintainer line (D.ShanB) with dissi cyto source and fell into cluster 3. It had short plant stature with short flag leaf but had high spikelet fertility and thousand grain weight. Early maturing group contained maintainer lines having 4 different cyto sources revealed early maturing group featured with cyto source variation with high panicle number $/ \mathrm{m}^{2}$ and very good yield potentiality. Grouping the maintainer lines on the basis of maturity period yield per plot was proportionally higher in late maturing line. Only a single line Gan46B fell into late maturing group and had highest yield. This fact suggests possibility of corresponding CMS line of Gan46B could be used as female if restorer lines are selected from early maturing group. Medium maturing group had some added advantage like medium duration with diverse cyto sources and good yielding ability. If restorer lines are selected from late maturing group with high yield potentiality proper hybrid rice combination become available for Boro season and it would be better because rice hybrid perform better under irrigated Boro eco system. One hundrade and nineteen days growth duration also very good for T. Aman season.
\end{abstract}

Keywords: Genetic diversity; maintainer lines; morphological traits; maturity

DOI: http://dx.doi.org/10.3329/bjpbg.v28i1.27844

\section{INTRODUCTION}

Rice has special position as a source of providing over $75 \%$ of Asian population and more than three billion of world populations meal which represents 50 to $80 \%$ of their daily calorie intake (Khush, 2005; Amirjani, 2011). This population will increase to over 4.6 billion by 2050 (Honarnejad et al., 2000) which demands more than $50 \%$ of rice needs to be produced what is produced present to cope with the growing population (Ashikari et al., 2005; Srividya et al., 2010). Yields of improved rice varieties in favorable conditions have reached a plateau or even subsequently declined in many 
countries including Bangladesh. To meet the challenge of producing more rice under those constraints, we need new technologies like hybrid rice because it gives 15-30\% yield advantage over inbred rice. Moreover, hybrid rice has also shown better performance under adverse conditions like drought and saline conditions (FAO, 2004). Increasing demand for rice especially in developing countries hybrid rice is considered as a viable alternative technology for breaking the present yield ceiling of modern varieties. So far 108 hybrid rice varieties released from National Seed Board under various private companies and public organization (SCA, 2012). Majority of these hybrids are imported from China. In Boro 2011-2012 seasons, total sold rice hybrid was 8800 MT of which $45 \%$ were imported from China and the rest $55 \%$ were from local production (Huda and Ali, 2012). If we develop or improve parental lines of hybrid rice based on diversity we can find most diverse parents that will help developing heterotic hybrid combinations. Such a way we can save lots of foreign currency and make a remarkable impact on our national economy. This study will help in selection of more distantly related maintainer lines for using in crossing program and ultimately help to develop high yielding hybrid rice varieties in local conditions. So, the knowledge of diversity among parental lines is very much important. With this view, this study was undertaken to evaluate the maintainer lines based on cluster analysis and days to maturity.

\section{MATERIALS AND METHODS}

The experiment was conducted at the experimental field of Bangladesh Rice Research Institute (BRRI), Gazipur during T. Aman 2007 season. Ten maintainer lines were evaluated to identify most diverse maintainer lines in respect of morphological traits for developing heterotic rice hybrids based on clustering and days to maturity. Twenty one days old single seedling was transplanted with a spacing of $20 \mathrm{~cm} \mathrm{x} 15 \mathrm{~cm}$ in RCB design with three replications. The unit plot size was $1.8 \mathrm{~m} \times 1.0 \mathrm{~m}$. Adequate soil fertility was ensured by applying Urea-TSP-MP-Gypsum-ZnSO ${ }_{4} @$ 150:100:70:60:10 $\mathrm{kg} / \mathrm{ha}$, respectively. Total amount of TSP, MP, Gypsum and $\mathrm{ZnSO}_{4}$ were applied during final land preparation. The urea was applied in three installments at 15 days after transplanting (DAT), 30 DAT and 45 DAT. Necessary intercultural operations was carried out during cropping period for proper growth and development of the plants. Ten sample plants were randomly selected from each plot excluding the border plants and data were recorded on plant height $(\mathrm{cm})$, days to $50 \%$ flowering, flag leaf length $(\mathrm{cm})$, number of panicles $/ \mathrm{m}^{2}$, spikelet fertility (\%), panicle weight $(\mathrm{g})$, days to maturity, 1000 grain weight $(\mathrm{g})$ and grain yield/plot $(\mathrm{kg})$. List of maintainer lines along with cyto-source and country of origin are given in Table 1.

Table 1. List of maintainer lines used in this experiment

\begin{tabular}{c|l|l|l}
\hline Sl.No. & Designation & Cytosource of corresponding CMS lines & \multicolumn{1}{c}{ Country of origin } \\
\hline 01 & IR58025B & Wild abortive (WA) & Philippines \\
02 & II32B & Indonesian paddy (ID) & China \\
03 & Jin23B & Wild abortive (WA) & Philippines \\
04 & IR78362B & Dissi & Philippines \\
05 & D.ShanB & Dissi & China \\
06 & Gan46B & Gambiaca & China \\
07 & BRRI3B & Wild abortive (WA) & Bangladesh \\
08 & BRRI9B & Gambiaca & Bangladesh \\
09 & IR75608B & Dissi & Philippines
\end{tabular}




\begin{abstract}
10 IR77811B Kalinga Philippines
Euclidean distance

Euclidean or straight-line measure of distance is the most commonly use statistic for estimating genetic distance (GD) between individuals (genotypes or populations) by morphological data. Euclidean distance between two individuals $i$ and $j$, having observations on morphological characters $(p)$ denoted by $x_{1}, x_{2}, \ldots, x_{p}$ and $y_{1}, y_{2}, \ldots, y_{p}$ for $i$ and $j$, respectively, can be calculated by the following formula (Minitab software, 2010): $\quad d(i, j)=\left[\left(x_{1}-y_{1}\right)^{2}-\left(x_{2}-y_{2}\right)^{2}+\ldots\left(x_{p}-y_{p}\right)^{2}\right]^{1 / 2}$

The centroid is the middle of a cluster. It is a vector containing one number for each variable, where each number is the mean of a variable for the observations in that cluster. The centroid was used as a measure of cluster location. For a given cluster, the average distance from the centroid was the average of the distances between observations and the cluster centroid. The maximum distance from the centroid was the maximum of these distances. It was measured using Minitab statistical software, Minitab Inc. USA. Dendrogram showing the dissimilarity in 10 maintainer lines for days to maturity is shown in Figure 1.
\end{abstract}

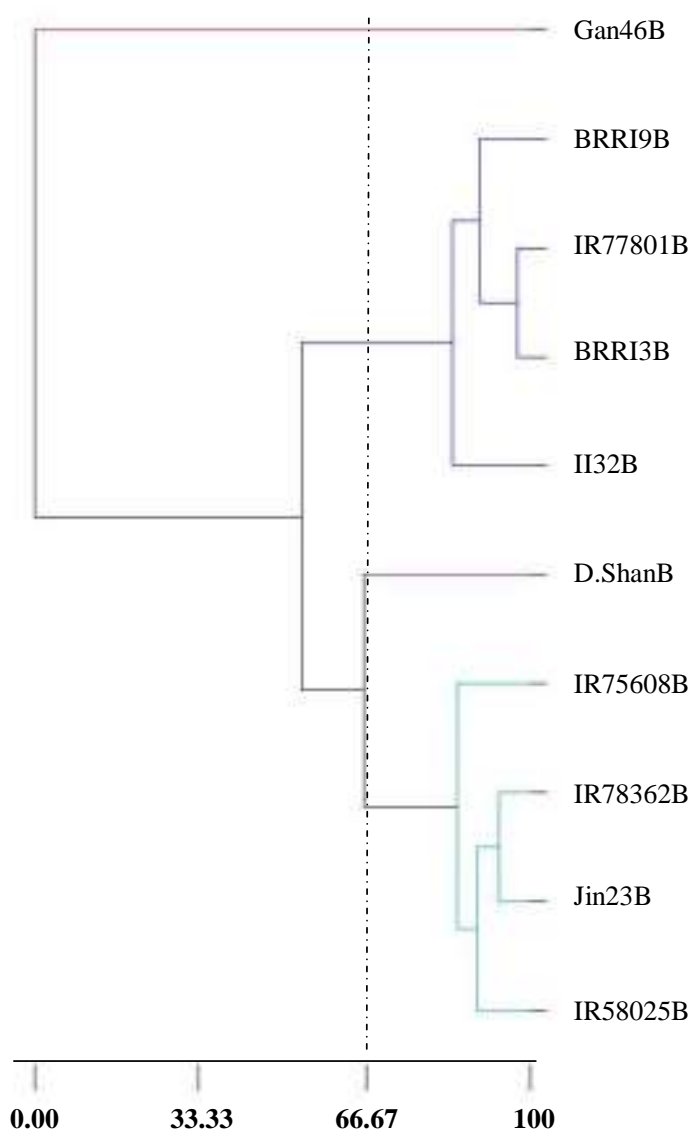

Figure 1: Dendrogram showing the dissimilarity in 10 maintainer lines for days to maturity 


\section{RESULTS AND DISCUSSION}

Based on mean values of different morphological traits, ten (10) maintainer lines were classified into four distinct clusters (Table 2). Cluster 1 contained four maintainer lines of which two had different cyto sources of CMS in their corresponding A lines. Viz. Wild abortive (IR58025B and Jin23B) and dissi cyto source (IR78362B and IR75608B) and fell into early and medium maturing group. Maximum distance of cluster 1 from centroid was 1.85 . Cluster 2 contained four maintainer lines having four different cyto sources of CMS in their corresponding A lines. They were Indonesian paddy (ID) type (II32B), wild abortive (BRRI3B), Kalinga (IR77811B) and gambiaca (BRRI9B) and fell into early and medium maturing group.

Table 2. Grouping result of 10 maintainer lines of rice

\begin{tabular}{l|l|c|c}
\hline Group & \multicolumn{1}{c|}{ Maintainer lines } & Number of lines & Fall in cluster \\
\hline Very early & D.ShanB & 1 & 3 \\
Early & Jin23B, IR78362B, IR75608B & 3 & 1 \\
& II32B & 1 & 2 \\
Medium & IR58025B & 1 & 1 \\
\multirow{2}{*}{ Late } & BRRI9B, IR77811B, BRRI3B & 3 & 2 \\
\hline
\end{tabular}

Table 3. Different statistics of Euclidean distance and cluster analysis of the maintainer lines

\begin{tabular}{c|c|c|c|c}
\hline Cluster & No. of lines & $\begin{array}{c}\text { Within cluster sum } \\
\text { of squares }\end{array}$ & $\begin{array}{c}\text { Average distance } \\
\text { from centroids }\end{array}$ & $\begin{array}{c}\text { Maximum distance } \\
\text { from centroids }\end{array}$ \\
\hline Cluster1 & 4 & 10.46 & 1.60 & 1.85 \\
Cluster2 & 4 & 11.46 & 1.64 & 2.13 \\
Cluster3 & 1 & 0.00 & 0.00 & 0.00 \\
Cluster4 & 1 & 0.00 & 0.00 & 0.00 \\
\hline
\end{tabular}

Table 4. Distances between cluster centroids in 10 maintainer lines of rice

\begin{tabular}{l|c|c|c|c}
\hline & Cluster 1 & Cluster 2 & Cluster 3 & Cluster 4 \\
\hline Cluster1 & 0 & & & \\
Cluster2 & 3.94 & 0 & & \\
Cluster3 & 4.14 & 4.36 & 0 & \\
Cluster4 & 6.69 & 5.59 & 8.61 & 0 \\
\hline
\end{tabular}

On the other hand cluster 3 contained one maintainer line from dissi cyto source (D.ShanB) and belonged to very early maturing group. On the contrary, cluster 4 contained one maintainer line from gambiaca cyto source (Gan46B) and belonged to late maturing group. Maximum distance of cluster 2 from centroids was 2.13 whereas cluster 3 and 4 were found in the centroids (Table 3 ).

Distance between cluster centroids ranged from 3.94 to 8.61 (Table 4). The lowest distance between cluster centroids was found in the cluster 1 and cluster 2 (3.94) and the highest was found in the cluster 3 and cluster 4 (8.61). It indicated that the lines fell into cluster 1 and 2 had some genetic similarity and thus they were not very far from centroids. On the contrary, due to genetic dissimilarity the line fell into cluster 3 and 4 had maximum distances from centroids. In this study, most of the maintainer lines were 
grouped into early and medium maturing clusters while the other two lines were clustered into very early and late maturing cluster (Table 2). D. ShanB from cluster 3 had short plant stature with short flag leaf but had high spikelet fertility and thousand grain weight. Early maturing group contained maintainer lines having 4 different cyto sources. So, early maturing group featured with cyto source variation with high panicle number $/ \mathrm{m}^{2}$ and very good yield potentiality. So, proper combination of high yielding restorer lines with corresponding CMS line could offer potential rice hybrid for transplanted Aman season. Maurya and Singh (1977) reported that maturity time, plant height and number of productive tillers contributed most to the divergence in rice. Similar sorts of results also reported by Julfiquar et al. (1985) while studying diversity with some maintainer and restorer lines of rice. Medium maturing group contained maintainer lines from 3 different cyto sources with high spikelet fertility and good yield potentiality. Late maturing group contained very long flag leaf, high panicle number $/ \mathrm{m}^{2}$, heavy panicle weight, maximum grains/panicle with highest yield potentiality. Grouping the maintainer lines on the basis of maturity period yield per plot was proportionally higher in late maturing line (Table 5).

Table 5. Mean values for yield and yield contributing characters within four maturing groups

\begin{tabular}{l|c|c|c|c|c|c|c|c|c|c|c}
\hline \multicolumn{1}{c}{ Group } & $\begin{array}{c}\text { PHT } \\
(\mathrm{cm})\end{array}$ & $\begin{array}{c}\text { N/ } \\
\mathrm{T}\end{array}$ & $\begin{array}{c}\text { D50\%F } \\
(\text { days })\end{array}$ & $\begin{array}{c}\text { FLL } \\
(\mathrm{cm})\end{array}$ & $\begin{array}{c}\text { Pan } \\
/ \mathrm{m}^{2}\end{array}$ & $\begin{array}{c}\text { PW } \\
(\mathrm{g})\end{array}$ & $\begin{array}{c}\text { SF } \\
(\%)\end{array}$ & $\begin{array}{c}\text { grains } \\
/ \mathrm{pan}\end{array}$ & $\begin{array}{c}\text { DTM } \\
(\text { days })\end{array}$ & $\begin{array}{c}1000 \\
\text { GW }(\mathrm{g})\end{array}$ & $\begin{array}{c}\text { Gy/ } \\
\text { Plot }(\mathrm{g})\end{array}$ \\
\hline Very early & 88.8 & 4.0 & 66.0 & 27.9 & 145.2 & 2.56 & 78.51 & 90.3 & 94.0 & 26.0 & 345.5 \\
Early & 101.9 & 5.1 & 75.3 & 32.5 & 173.8 & 2.57 & 67.94 & 97.2 & 105.0 & 20.7 & 403.9 \\
Medium & 112.3 & 4.3 & 83.3 & 31.9 & 139.4 & 3.20 & 77.20 & 97.4 & 110.3 & 23.5 & 439.6 \\
Late & 117.1 & 6.3 & 90.0 & 50.0 & 146.3 & 4.74 & 81.00 & 130.5 & 119.0 & 24.8 & 770.0 \\
\hline
\end{tabular}

$\mathrm{PHT}=$ Plant height; N/T= No. of effective tillers; $\mathrm{D} 50 \% \mathrm{~F}=$ Days to $50 \%$ flowering; FLL= Flag leaf length; No.pan $/ \mathrm{m}^{2}=$ No. of panicle $/ \mathrm{m}^{2} ; \mathrm{PW}=$ Panicle weight; SF\%= Spikelet fertility; Grains/pan= Number of grains/panicle; DTM= Days to maturity; $1000 \mathrm{GW}=1000$ grain weight; Gy/plot= Grain yield/plot

Only a single line Gan46B fell into late maturing group but its yield was much higher than the lines included in very early, early and medium maturing group. This line was included in cluster 4 which exhibited highest distance from other clusters. Moreover, other yield contributing characters indicated its high yield potentiality. This facts suggest possibility of corresponding CMS line of Gan46B could be used as female if restorer lines are selected from early maturing group. But actually early maturing restorer line had low fertility restoration ability. So, it is very difficult to find potential hybrid combination from this group. If we consider medium maturing group, it had some added advantage like medium duration with diverse cyto sources and good yielding ability. If restorer lines are selected from late maturing group with high yield potentiality proper hybrid rice combination become available for Boro season and it would be better because rice hybrid perform better under irrigated Boro eco system.

Sheng-jun et al. (2006) found in a study 15 maintainer lines in early or mediummaturing cluster, 4 restorer lines in early-maturing and in case of thermo-sensitive genic male sterile 16 restorer lines in medium or late-maturing cluster and 4 maintainer lines in medium or late-maturing cluster. Yu et al. (2005) studied the genetic variation in rice varieties derived from Aizizhan using morphological traits and their cluster analysis showed the difference in genetic distance among varieties determined by morphological traits. Only those elite lines that are diverse from each other will be hybridized to 
develop a new hybrid variety and could maximize opportunities to obtain superior hybrids because unrelated parents would be expected to contribute unique desirable alleles at different loci (Tatineni et al., 1996). The selection of diverge genotype from cluster would produce a broad spectrum of variability for morphological and quality traits studied which may enable further selection and improvement. The hybrid developed from the selected genotypes within the limits of compatibility of these clusters may produce high magnitude of heterosis. This would be rewarding in hybrid rice breeding programme. Sarawgi and Shrivastava (1996), Sarawgi and Rastogi (2000), Roy et al. (2002) and Naik et al. (2004) were in agreement with these findings.

Genetic diversity is fundamentally important for developing heterotic rice hybrid. Results of this study indicated that maintainer lines are diversed. Among the maintainer lines medium maturing group and late maturing group have diverse cyto sources and good yielding ability. If restorer lines are selected from late maturing group with high yield potentiality that will predict high yield heterosis with desire growth duration.

\section{ACKNOWLEDGEMENTS}

This work was supported by Ministry of Agriculture, Bangladesh through a project "Research and Development of Hybrid Rice in Bangladesh".

\section{REFERENCES}

Amirjani, M. R. 2011. Effect of salinity stress on growth, sugar content, pigments and enzyme activity of rice. International Journal of Botany 7 (1): 73-81.

Ashikari, M., H. Sakakibara, S. Lin, T. Yamamoto and T. Takashi. 2005. Cytokinin oxidase regulates rice grain production. Science 309: 741-745.

FAO. 2004. Hybrid rice for food security. http://www.rice 2004.org.

Honarnejad, R., S. Abdollahi, M. S. Mohammad-Salehi and H. Dorosti. 2000. Consideration of adaptability and stability of grain yield of progressive rice (Oryza sativa L.) lines. Research Agriculture Science 1: 1-9.

Huda, N. and Shahjahan Ali, 2012. Hybrid rice seed technology in Bangladesh. Issues and Considerations. Seminar presented at BARC, Farm gate, Dhaka on September 18, 2012

Julfiquar, A. W., S. S. Virmani and A. L. Carpena. 1985. Genetic divergence among some maintainer and restorer lines in relation to hybrid breeding in rice (Oryza sativa L.). Theoretical and Applied Genetics 70: 671-678.

Khush, G. S. 2005. What it will take to feed 5.0 billion rice consumers in 2030. Plant Molecular Biology 59: 1-6.

Maurya, D. M. and D. P. Singh. 1977. Genetic divergence in rice. Indian Journal of Genetics and Plant Breeding 37: 394-402.

Minitab software. 2010. Minitab statistical software, Minitab Inc. USA. Version, 2010.

Naik, A. R., D. Chaudhury and J. N. Reddy. 2004. Genetic divergence studies in scented rice. Oryza 40: 79-82.

Roy, B., A. K. Basu and A. B. Mandal. 2002. Genetic diversity in rice (Oryza saliva L.) genotypes under humid tropics of Andamam based on grain yield and seed charecters. Indian Journal of Agricultural Science 72: 84-87.

Sarawgi, A. K. and M. N. Shrivastava. 1996. Genetic divergence in rice under irrigated and rainfed condition. Advances Plant Science 9: 93-100. 
Sarawgi, A. K. and N. K. Rastogi. 2000. Genetic diversity in traditional aromatic rice accessions from Madhya Pradesh. Indian Journal of Plant Genetics Research 13: 138-146.

Seed Certification Agency 2012. List of released hybrid rice varieties from 1997 to 2011.

Sheng-jun, W., L. Zuo-mei and W. Jian-min. 2006. Genetic diversity among parents of hybrid rice based on cluster analysis of morphological trait and simple sequence repeat markers. Rice Science 13: 155-160.

Srividya, A., L. R. Vemireddy, A. S. Hariprasad, M. Jayaprada and S. Sridhar. 2010. Identification and mapping of landrace derived QTL associated with yield and its components in rice under different nitrogen levels and environments. International Journal of Plant Breeding and Genetics 4: 210-227.

Tatineni, V., R. G. Cantrell and D. D. Davis. 1996. Genetic diversity in elite cotton germplasm determined by morphological characteristics and RAPDs. Crop Science 36: 186-192.

Yu, H. Y., X. H. Wei, Y. P. Wang, X. P. Yuan and S. X. Tang. 2004. Study on genetic variation of rice varieties derived from Aizizhan by using morphological traits, allozymes and simple sequence repeat (SSR) markers. Chinese Journal Rice Science 18: 477-482. 